Linguistique, littérature, didactique

\title{
Les livrets pour enfants dans les musées d'art : vers une médiation culturelle et récréative
}

Art Museum guides for children: toward a cultural and antertainment mediation

\section{Françoise Rigat}

\section{(2) OpenEdition}

\section{Journals}

Édition électronique

URL : http://journals.openedition.org/pratiques/3707

DOI : $10.4000 /$ pratiques.3707

ISSN : 2425-2042

Éditeur

Centre de recherche sur les médiations (CREM)

Référence électronique

Françoise Rigat, "Les livrets pour enfants dans les musées d'art : vers une médiation culturelle et récréative », Pratiques [En ligne], 175-176 | 2017, mis en ligne le 22 décembre 2017, consulté le 14 novembre 2019. URL : http://journals.openedition.org/pratiques/3707 ; DOI : 10.4000/pratiques.3707

Ce document a été généré automatiquement le 14 novembre 2019.

(c) Tous droits réservés 


\title{
Les livrets pour enfants dans les musées d'art : vers une médiation culturelle et récréative
}

Art Museum guides for children: toward a cultural and antertainment mediation

\author{
Françoise Rigat
}

\section{Présentation et problématiques du livret pour enfants}

\section{Contexte et attentes de la visite en famille}

1 Aujourd'hui, la plupart des institutions muséales proposent des livrets spécifiquement destinés aux jeunes visiteurs pour leur permettre de découvrir l'exposition et ses contenus en autonomie. Plus récemment, un nouveau support de visite est venu enrichir l'éventail des textes enfants: les livrets ciblant l'enfant et les adultes accompagnateurs (famille). L'offre de tels documents coïncide d'une part, avec une demande réelle de la part des familles d'outils qui puissent favoriser l'appropriation de l'exposition par chacun ${ }^{1}$ et, d'autre part, avec l'augmentation notable de la fréquentation de cette nouvelle catégorie de visiteurs, dans la majorité peu formée.

D'après J.Eidelman et A. Jonchery (2011), l'expansion du «tourisme culturel populaire » va de pair avec les changements importants survenus dans les modes de relation à la culture, où la sphère des loisirs est de plus en plus culturalisée, et le partage entre établissements muséaux et lieux de consommation culturelle de moins en moins certain (Chaumier, 2011) : il suffira de citer, à titre d'exemples, la toute dernière exposition au Louvre ${ }^{2}$, dans laquelle la statue d'Hercule du XVII siècle côtoie le masque de Dark Vador, ou encore l'invasion des Pokémons au Quai Branly, le jeu de l'été !

Dans ce contexte, les objectifs parentaux se sont quelque peu modifiés : s'ils s'affirment toujours en termes d'éducation au sens large, c'est-à-dire de sensibilisation à l'art et de développement personnel de l'enfant (Jonchéry \& Biraud, 2014), la visite se conçoit désormais comme un moment de détente, de partage (Eidelman \& Jonchery, 2011), 
voire de divertissement (Chaumier, 2011) si l'on en croit le succès des applications mobiles muséales (Lesaffre, Watremez \& Flon, 2014).

4 Ces mutations dans les modes de visite, et le profil particulier du public familial que l'on notait à l'instant, nous font revenir sur le rôle et la conception des livrets pour enfants (Rigat, 2012): comment s'inscrivent-ils dans les liens nouveaux entre les pratiques de visite et de loisir ? C'est ce point que l'on voudrait précisément interroger, à partir de l'examen critique de quelques livrets, qu'ils soient destinés à l'enfant seul ou à toute la famille, et des formes de médiation qu'ils construisent.

\section{D'une médiation éducative à une médiation culturelle}

Avant d'entrer dans le vif du sujet, il parait opportun d'insister sur ce qui fait du livret un outil de culture ${ }^{3}$. Cette proposition, apparemment simple, demande en réalité à être précisée.

On a coutume de définir le musée comme lieu d'éducation non formelle (Jacobi \& Coppey, 1995, p. 12) ${ }^{4}$, et plus récemment, comme lieu culturel de formation (Le Marec, 2011). Cette évolution n'est pas anodine. On voit en effet se dessiner des convergences de vues parmi les chercheurs d'horizons différents sur une conception plus culturelle de l'éducation entendue au sens de "comprendre et ressentir plutôt qu'au sens de faire apprendre et vulgariser ", comme le souligne M.-S. Poli (2013, p. 168). Celle-ci dépasse le pédagogique stricto sensu pour englober les valeurs symboliques et sociales telles que, par exemple, la transmission des valeurs du musée et l'initiation à l'usage de la visite (Jacobi, Meunier \& Romano, 2000, p. 52). N'est-ce pas au fond ce qui permet de plaider pour la singularité du musée par rapport à l'école?

7 Et cependant, dans la pratique, le livret tend souvent à confondre l'éducation et l'instruction, se réduisant à un empilement de connaissances: biographies et chronologies exhaustives, listes de noms propres et de données factuelles, exercices de vocabulaire, glossaires, compréhensions de textes, etc. C'est en tout cas ce que l'on a pu constater en feuilletant nombre de livrets proposés: parce qu'ils circonscrivent l'acculturation à la transmission de connaissances, ils n'exploitent pas vraiment les potentialités éducatives au sens large de la visite comme le prônent les chercheurs (Jacobi, Meunier \& Romano, 2000). Or, c'est précisément contre cette vision du rapport au savoir que le champ de la médiation culturelle s'est construit, dans une visée plus démocratique du partage de la culture (Desvallées \& Mairesse, 2011). Enfin, autre point crucial : cette position culturelle permet de sortir la visite de la dichotomie, désormais obsolète et maintes fois dénoncée, entre éducation et divertissement et de la rapprocher du «loisir éducatif» (l'edutainment d'outre-Manche), du plaisir lié à la culture, à l'expérience, à la sociabilité - pas seulement à la contemplation donc.

8 Mais comment le livret peut-il glisser d'une démarche éducative à un mode culturel ? C'est la question que posaient déjà D. Jacobi et O. Coppey en 1995, ce qui prouve que la tâche est plus complexe qu'elle ne parait, ne serait-ce que parce qu'il n'y va pas de la même éthique culturelle pour toutes les institutions.

\section{L'ancrage institutionnel du livret}

Comme toute pratique de médiation, la conception du document est fortement institutionnalisée : elle soutient en effet des logiques liées à l'identité historique et 
politique, aux représentations, aux valeurs propres à chaque institution aussi bien qu'à l'autorité que celle-ci exerce, à sa position dans le paysage culturel français - sans oublier le budget et la compétence particulière de l'équipe de médiateurs. Le projet éducatif et culturel n'y est donc pas pareillement partagé selon l'institution. Ainsi, certains musées qui ont le label « Musée de France ", tel que le Louvre, et pour lesquels le public est au centre de "l'effort de transmission des savants aux ignorants " (Le Marec, 2006), ont des fins ouvertement éducatives, alors que pour d'autres, l'éducation n'est à priori qu'un aspect partiel: les centres d'art contemporains, par exemple, focalisent leurs enjeux sur des actions aptes à diffuser et dynamiser la scène artistique, et relèvent de logiques plus communicationnelles qu'éducatives.

Il en est de même pour les collections, différemment mobilisées dans les musées des beaux-arts et les centres d'art contemporain parce que renvoyant à des modes spécifiques de présentation (Gob \& Montpetit, 2010) : plus l'art est classique, plus la démarche proposée est contemplative, plus le discours est historique et esthétique; à l'opposé, la création contemporaine, souvent conceptuelle, immatérielle et performative, réclame des explications qui d'évidence s'éloignent de l'histoire de l'art. C'est pourquoi les choix explicatifs et interprétatifs que développe chaque institution sont aussi hérités de traditions, de modèles, de valeurs qui les légitiment et les rendent finalement prévisibles: un enrichissement de la connaissance pour les musées des beaux-arts vs un enrichissement de l'expérience pour ceux d'art contemporain.

\section{La question du destinataire enfant}

11 Le destinataire pose différents problèmes et en premier lieu celui de l'étendue d'âge recouvert par l'hyperonyme enfant ${ }^{5}$. En principe, on entend par enfant les 8-12 ans ${ }^{6}$ et par jeune les pré-adolescents ou les adolescents. Or l'univers émotionnel de l'enfant ou $\mathrm{du}$ jeune, ses capacités cognitives et interactives sont d'une nature éminemment variable et complexe : l'expérience magistrale enseigne en effet que l'enfant, à cet «âge transitoire de la vie», comme le définit joliment la Convention des Nations Unies relative aux droits de l'enfant, est «aussi instable qu'insaisissable » ${ }^{7}$. Pour preuve : la plupart des documents n'inscrivent pas l'âge ciblé dans le paratexte. De ce point de vue, sans doute est-il plus pratique de s'adresser à un écolier, un collégien ou un lycéen, dont les compétences et les connaissances attendues sont fixées par cycles d'étude et définies par les programmes ministériels, qu'à un enfant ou un jeune. Sans compter que la famille en visite au musée se compose souvent de garçons et filles d'âge différent ${ }^{8}$.

12 Plus généralement, si aujourd'hui on s'accorde pour en faire des personnes à part entière (Octobre \& Sirota, 2013, p. 19), ils restent des adonaissants, en construction d'une identité individuelle qui surgit à la lumière d'un milieu social, de choix éducatifs faits par la famille comme l'ont enseigné les travaux de P. Bourdieu, mais aussi d'une culture enfantine ou jeune commune, sociale et distincte (Wilfried, 2007).

13 Enfin, les documents posent la question du rôle de l'adulte accompagnateur, en particulier dans le cas du livret pour la famille : qui de l'enfant ou de l'adulte assume le rôle de médiateur, c'est-à-dire fait le lien entre l'œuvre et l'accompagnant ${ }^{9}$ ? 


\section{Choix du corpus d'analyse}

Au départ, on a consulté une cinquantaine de livrets environ, recueillis sur place, au gré de nos visites, ou téléchargés sur les sites web des musées, que l'on a finalement ramenés à cinq pour cette étude. En effet, on a sélectionné des livrets très différents afin de rendre compte, par une caractérisation contrastive, des voies plurielles qu' adopte ou peut adopter la médiation envers les enfants.

Par conséquent, sans prétendre nullement à l'exhaustivité, on a retenu des livrets pour leur caractère tantôt ingénieux, tantôt gai et hardi, voire audacieux, mais toujours exigeant ; ensuite, on a considéré, autant que faire se peut, la variété des facteurs suscités et choisi des livrets destinés à des enfants d'un âge différent (enfant et préadolescent), provenant de contextes muséaux connus et moins connus, avec ou sans collection (musée national, fondation et centre d'art), et d'expositions de diverses sortes (monographique, thématique) commentant des œuvres d'art aussi bien anciennes que modernes et contemporaines.

Le présent travail abordera les cinq livrets successivement: le premier s'inscrivant dans une approche pédagogique classique, afin de mieux repérer les filiations et les ruptures des trois livrets ultérieurement analysés, le dernier étant destiné à la famille, ce qui permettra de retrouver certaines questions évoquées dans les documents précédents. Le point de vue que l'on adoptera est discursif et énonciatif, avec pour axe de réflexion la question soulevée par É. Caillet (1994) : qu'est-ce qui se construit entre les œuvres, l'enfant et l'adulte et, plus largement, entre l'enfant et le musée ? En conclusion, on évoquera un questionnement bien ancien, mais loin d'être résolu, sur la démocratisation de l'art (Glicenstein, 2012) que ces documents suggèrent.

\section{Un modèle pédagogique prégnant : « le tête-à-tête sensible et informé ${ }^{10}$ de l'enfant avec l'œuvre}

\section{Un espace et un parcours de lecture contraignants}

17 Pour illustrer l'idée bien établie selon laquelle il faut apprendre à l'enfant à voir, on s'en tiendra à un exemple unique, le Parcours enfant "Trop grand!» publié par le Louvre, archétype de l'approche traditionnelle. Il s'agit d'un dépliant de format carré, au papier brillant de qualité, joliment coloré et aéré, et d'une typographie sobre et facile à lire. La lecture recto-verso, en double orientation, et les nombreux volets en pli roulé le rendent cependant peu maniable. Les commentaires, non justifiés à droite, positionnés près des reproductions des statues, sont distribués autour d'un tracé épais qui matérialise un parcours fléché pourvu d'un "départ», de sept haltes autour d'œuvres ayant comme fil conducteur thématique la grandeur et, enfin, d'une " arrivée ». Il contient également un plan pour aider l'enfant à « se repérer » et à situer "les étapes de [s]a promenade ». En effet, la configuration labyrinthique bien connue du musée et le nombre des visiteurs nécessitent une gestion méticuleuse du déplacement physique :

[1] Prends le passage à droite des Captifs, puis tourne à gauche. Monte les marches puis les escaliers mécaniques jusqu'au rez-de-chaussée et entre dans le département des antiquités orientales. Traverse la salle 1, puis tourne à gauche dans la salle 2 et rends-toi dans la salle 4 . 

c'est plutôt une coquetterie linguistique ${ }^{12}$. Certes, l'enfant en retrouve la lenteur et la légèreté lorsqu'il se laisse aller à la libre jouissance des "monuments exceptionnels " qu'il « croise » (voir exemple [8] plus bas), à admirer la vue (« Regarde au sommet de l'escalier »), à la rêverie (« Essaie d'imaginer la hauteur d'une colonne ») mais c'est bien d'un parcours qu'il s'agit, tel qu'annoncé d'entrée de jeu dans le titre, et régulièrement rappelé comme dans ce passage typique des livrets d'art (« voici ce que tu vas voir ») :

[2] Ton parcours

Tu vas découvrir des peintures et des objets d'art... et donc parcourir une partie du musée.

ou lorsqu'il en éprouve les accidents (« Passe entre les deux lamassou»), les difficultés et enfin la gravité ("Essaie de retrouver les objets du sacré»). Bien que dans son sens métaphorique de cheminement moral, intellectuel, voire spirituel ${ }^{13}$, le parcours renoue avec la promenade, il s'en éloigne par sa connotation pédagogique. L'exemple ci-dessus préfigure un espace à traverser, non à investir : un espace dont le déroulement et le terme sont fixés ("Ta visite est maintenant terminée »), tout comme la finalité qui apparait nettement ici :

[3] Lis à voix basse les questions, commentaires et explications qui sont là pour t'aider à

regarder et donc à comprendre.

reconnaitra les éléments qui desservent une relation pédagogique et didactique traditionnelle : tout d'abord, la consigne, qui invite à une lecture silencieuse, solitaire, intime du livret et inculque la retenue; la modalité de présentation ensuite (la transmission au moyen de "questions/explications»); le savoir enfin, de tradition humaniste ${ }^{14}$, qui passe essentiellement par l'apprentissage de la perception visuelle. Ces trois caractéristiques ont pour conséquence une troisième: elles enferment l'enfant dans un rôle d'apprenti contemplateur/amateur dont l'autonomie est supposée acquise.

\section{L'apprentissage perceptif : « voir pour comprendre »}

21 Les œuvres sélectionnées, toutes significatives et hautement symboliques, donnent à voir un récit multimillénaire de l'Histoire de l'art ; les paragraphes se succèdent comme des séquences spatio-temporelles qui embrassent et unifient le monde : le lointain (la Syrie avec le palais du roi Sargon II, le royaume perse de Darius, l'Égypte avec le sarcophage de Ramsès III, la Grèce de la victoire de Samothrace), le proche (le Louvre, le roi soleil, Napoléon) et des références précises qui comportent sans doute, en filigrane, le lecteur ciblé (un jeune parisien ?) :

[4] Ce groupe occupait autrefois le centre de la place des Victoires à Paris.

Les commentaires, dans leur nécessaire dépouillement descriptif, engagent prioritairement une activité d'observation de la dimension plastique. C'est pourquoi l'attention de l'enfant y est à chaque fois sollicitée sur un ton injonctif par le champ lexical de la vue:

[5] Observe la diversité de leurs expressions et note les signes de leur défaite : armes brisées, fers aux poignets...

[6] Approche-toi. Comment le sculpteur a-t-il suggéré la force du vent? Observe son corps. 

D’abord, celui-ci est orienté en fonction d'objectifs éducatifs: la première activité permet de reconnaitre et de rappeler les œuvres précédemment présentées; la seconde
éprouve la maitrise technique du dessin (respect des échelles, des proportions, de la symétrie). Il est clair ici que le «jeu» cherche moins à amuser qu'à renforcer un apprentissage, vérifier des connaissances nouvellement acquises. Ensuite, on constate l'absence des dimensions subjective et émotionnelle typiques du jeu, puisque celui-ci n'est pas utilisé comme moyen d'expression personnelle et créative (du moins ici), mais comme outil à la mémorisation et l'imitation. Or comme le rappelle C. Barbieux (2011), «les enfants jouent d'abord pour le plaisir qu'ils vont en retirer et non pas pour optimiser un quelconque apprentissage ». Du coup, ce «jeu», aussi avisé et plaisant soit-il, est susceptible de déjouer les promesses dont l'étiquette est porteuse («Amusetoi »).

\section{Les représentations de l'écrit}

29 Le modèle pédagogique définit aussi les rapports énonciatifs. Ici, il y a bien une instance racontante qui s'adresse à l'enfant via le tutoiement, mais c'est une voix hors champ, par en haut: une voix distante qu'accentue le ton injonctif, l'absence de 
déictique et de pathos (à l'exception des phrases exclamatives). Cette impression est renforcée par l'emploi de la langue littéraire, sur le plan lexical (le registre soutenu, par exemple les car, en lieu et place du simple parce que) ainsi que sur le plan syntaxique (la question inversée, la pronominalisation, la nominalisation, les constructions adverbiale et détachée). Un seul exemple suffira :

[10] Le corps ne reposait pas à même la cuve.

Certes, il s'agit du Louvre ${ }^{15}$, du "musée des musées", où la langue soignée est en quelque sorte attendue ${ }^{16}$ mais en réalité, cette constatation vaut pour tous les livrets publiés par les musées nationaux et pour la plupart des musées des beaux-arts qui voient en lui un idéal pédagogique et discursif. Ainsi le musée d'art, bien connu comme prescripteur de culture, veille à maintenir la tradition sociale toute française de fonder les représentations de la langue sur l'écrit.

31 Quelles en sont les conséquences sur la conception culturelle du livret? Que d'une part, la langue soignée infléchit une «scolarisation » du livret : ce dernier se présente dans une certaine mesure comme un outil d'enrichissement de la langue, même si ce n'est pas sa vocation première. D'autre part, la culture est tout entière prise dans les programmes d'études du système scolaire : l'autodiscipline, le patrimoine mondial, la symbolique du pouvoir, la langue normée, etc. ce qui du coup rapproche fortement le musée de l'école. En conséquence de quoi, le bagage linguistique et encyclopédique de l'enfant pèse de tout son poids sur la compréhension du livret : si l'enfant ne maitrise pas les prérequis linguistiques ou culturels et si le musée inspire timidité et distance, le livret peut conduire à un rendez-vous manqué.

\section{Le ludique comme accès à l'œuvre : quand l'expérience esthétique devient une récréation}

\section{Rapprocher l'art de l'univers des jeunes}

À contrepied du parti pris précédent, le livret peut initier à l'art de manière décalée et humoristique. C'est la sensation que l'on a dès les premières pages du «Livret Jeune public » publié à l'occasion de l'exposition Les Papesses en Avignon :

[11] Tu commences ta visite aux portes de l'asile de Montfavet. Les deux gardiens de

l'entrée n'ont pas l'air super sympa... On n'y passerait pas nos vacances !

Ici aussi, la présentation est très plaisante : les pages aérées, les caractères ronds et fins, l'interlignage espacé, le texte non justifié à droite le rendent clair et agréable à lire mais là s'arrêtent les points communs avec le livret du Louvre. Contrairement au précédent en effet, celui-ci est long ( 32 pages) et très richement illustré (de 1 à 2 illustrations par page). Il s'en distingue aussi par l'âge du destinataire, puisqu'il est adressé à des lecteurs plus âgés, vraisemblablement une (pré)adolescente - on y reviendra - et surtout, par l'usage d'une langue décomplexée. À titre d'exemples :

[12] La dame en cire de l'artiste Berlinde De Bruyckere est plutôt flippante !

[13] Jane Sterbak défie la fashion police.

[14] Brrrr... On reste dans une ambiance qui donne la chair de poule !

Le registre familier, les anglicismes, la ponctuation expressive, les onomatopées, marquent un texte conversationnel, un discours au sens d'É. Benveniste.

[15] Berlinde de Bruyckere évoque ici le mythe d'Actéon, le célèbre chasseur.

Tu connais son histoire? 
Au cours d'une partie de chasse, il surprend la déesse Diane et ses copines nymphes en train de prendre leur bain. Furieuse qu'il les ai vues toutes nues, Diane le transforme en cerf.

Manque de pot, les propres chiens de chasse d'Actéon ne le reconnaissent pas et le dévorent tout cru!

Quel flair... Ils devaient être sacrément enrhumés ce jour-là !

Ci-dessus par exemple divers procédés concomitants installent une connivence avec le lecteur, qui est ainsi invité à partager le point de vue de l'énonciateur: "Pauvre Actéon! Mais au fond, il était bête ; bien fait pour lui ». Tous ces procédés donnent un style jubilatoire et vivace au texte. D'autres encore, tout aussi atypiques, comme les catégories émotives (les frissons, le truculent en [14]), et les références culturelles jeunes, puisées dans divers secteurs de la culture comme la littérature (Saint-Exupéry, Alice aux pays des merveilles), les fables (les contes d'Andersen), les dessins animés (Princesse Mononoké) sans oublier la musique (Madonna) et le cinéma, s'apparentent à ceux que l'on retrouve dans la littérature de jeunesse :

[16] On dirait que ce corps de mort-vivant est en train de se transformer en arbre,

comme les Ent dans Le Seigneurs (sic) des Anneaux.

Il s'agit de faciliter l'appropriation des œuvres : comme ci-dessus, en ramenant, par analogie (« on dirait») l'inconnu à ce que ce public connait bien, ou bien comme en [15], en échappant à l'écueil du didactisme ou de l'ennui que pourrait provoquer le récit mythologique.

\section{Le ludique, un vecteur de l'éducatif}

Il ne faut donc pas en déduire un appauvrissement des contenus, une gratuité du ludique. Ainsi, contrairement aux autres livrets, où ne figurent que des reproductions d'œuvres in praesentia, à vocation décorative ou mémorielle, l'image ici seconde l'interprétation puisqu'elle apporte un complément d'explication ${ }^{17}$, faisant par exemple figurer « l'original » d'une œuvre de K. Smith :

[17] Kiki Smith s'est inspirée d'un célèbre autoportrait de Frida Khalo pour réaliser cette œuvre. Regarde, ils ont la même posture!

L'air de rien, le ludique permet également de dédramatiser les œuvres plus terrifiantes (l'araignée géante de L. Bourgeois, les carcasses de B. de Bruyckere) et les sujets potentiellement traumatisants (le corps humain et ses tabous, tel que l'accouchement), comme ici :

[18] Oulala... ici l'ambiance se corse !

De grands meubles d'apothicaire enferment des formes mi-végétales mi-humaines...

$\mathrm{Tu}$ ne sais plus si tu es toujours dans la salle d'un musée ou dans la cachette d'un serial killer!

D'ailleurs, l'exposition semblerait peu conçue pour des enfants... Ceci expliquerait que le livret s'adresse à un jeune, et non à un enfant, et que certaines œuvres leur soient proscrites, comme le révèle ce commentaire amical, voire maternel ${ }^{18}$ :

[19] Tu retrouves ici une cellule de Louise Bourgeois mais cette fois-ci, ne va pas voir ce qui s'y passe à l'intérieur car tu risques de faire des cauchemars.

Quoi qu'il en soit, ces procédés énonciatifs et discursifs ont de nombreuses conséquences sur le plan symbolique, dans la mesure où le livret redistribue les usages de la culture et de la langue légitimes dans le musée. S'il est clair en effet qu'ils établissent une relation socioaffective avec le jeune public, répondant ainsi à une 
certaine fonctionnalité de la communication, ils aboutissent en fait à une vision défétichisée de l'art, à une image désacralisée du musée ${ }^{19}$. Ce qui importe, c'est de former un ludivisiteur ${ }^{20}$, d'apprivoiser les adolescents fâchés avec les musées, y compris les faibles lecteurs grâce à l'empan visuel réduit des paragraphes (les retours à la ligne après chaque phrase), à la brièveté de la phrase (deux lignes maximum), à la simplicité de l'enchainement parataxique.

41 Au final, ce livret constitue une alternative aux livrets traditionnels, didactiques et élitistes, en s'inscrivant ouvertement dans les tendances d'un nouveau rapport entre la culture savante et la culture ordinaire évoqué au début de cet article. Il représente également une tentative d'ouverture à d'autres champs du savoir, ce que l'on désigne par l'expression "médiation artistique et culturelle», souvent comprise - à tort comme « médiation artistique et cultivée ».

\section{L'art du côté des filles : les représentations de la culture jeune et du rapport du genre} exemple). Autrement dit, dans les mots de G. Brougère (2012), ce livret plutôt «fun » s'appuie sur des éléments "cute» (Disney pour s'entendre) tout en utilisant les caractéristiques du « cool » qui lui, relève de la culture adolescente (Spice girls). Encore faut-il ajouter que le cool résiste mal en général au temps qui passe (les Spice girls, justement)... L'effet de balancier entre des consommations culturelles et générationnelles diverses donne corps à l'hypothèse que la destinatrice visée du livret pourrait être aussi une "jeune adulte», cette fille délicieusement impertinente, curieuse, tendre, loufoque qui sommeille en chaque femme... 
Enfin, troisième constat: le récit anecdotique véhicule des représentations standardisées du monde féminin. Voici un exemple qui en interroge la pertinence :

[23] Ce sont les sculptures de la belle Camille Claudel qui occupent la première salle. Elle était le modèle et surtout l'amoureuse d'Auguste Rodin, un célèbre sculpteur. "Le penseur » de Rodin, ça te dit surement quelque chose... Leur histoire d'amour était loin d'être un long fleuve tranquille, mais cette passion dévorante inspirait constamment leur travail respectif. Tu vois le portrait de Camille Claudel par Rodin, très doux, et le portrait de Rodin par Camille, très imposant! [...] Camille Claudel sera délaissée par son amoureux et finira toute seule.

L'exaltation et l'émancipation féminine d'abord annoncée ("Ce n'est pas une exposition pour les fillettes!») s'étiolent devant la permanence de stéréotypes dans la vision de la femme artiste, qui reste présentée selon des traits traditionnels liés à la beauté, au milieu familial, à l'amour. Comme on le voit bien en [23], le récit pathétique des heurts et malheurs de "la belle Camille Claudel», associée à la déception amoureuse, à la fragilité toute féminine, à Rodin ("surtout»), finit par en minorer le génie artistique. Au bilan, on peut déplorer que ce texte original, à bien des égards subversif, ne soit pas allé jusqu'au bout pour battre en brèche le préjugé tenace sur l'art des femmes...

\section{De l'objet (d'art) au sujet (l'enfant) : du savoir voir au savoir être}

\section{D'une éducation à l'art à une éducation par l'art}

Le livret peut être une porte d'entrée pour découvrir l'autre, se découvrir, se confronter à un imaginaire et à un regard singulier - celui de l'artiste : telle est la démarche du Cahier découverte enfant, publié lors de l'exposition monographique de S. Blocher. Il s'agit d'un grand cahier papier (de format A4) de 6 pages, quelque peu encombrant pour être bien saisi par de petites mains et pour un confort de lecture ; la mise en page et la typographie sont soignées et bien lisibles, bien que la couleur rouge clair des caractères puisse se discuter.

Celui-ci commente un choix de neuf installations, dans l'ordre des salles. Le parcours de lecture est organisé uniformément: un titre simple en majuscule résumant ce que l'œuvre communique, un paragraphe explicatif plus ou moins long sur sa dimension plastique (ce qu'elle nous montre), et des activités interprétatives occupant les $3 / 4$ de la page (ce qu'elle suggère). Plusieurs encadrés intitulés «Le sais-tu?» et venant se positionner soit en bas du texte soit dans les marges, donnent un ton pédagogique au livret.

51 D'emblée, la définition succincte de l'art qui figure en préambule du livret - l'un des rares à en donner une, il faut le souligner - engage l'enfant dans une pratique subjective, puisqu'il y est défini en termes de «liberté » et d'« imaginaire », non d'esthétique :

[24] L'art est un espace de liberté dans lequel il est possible de se libérer des contraintes du quotidien et d'ouvrir son imaginaire.

52 À l'opposé des documents précédents, l'enfant n'est pas sollicité dans ce qu'il sait mais dans ce qu'il est. L'appréhension et la compréhension de l'œuvre ne se situent donc plus dans une logique du voir, mais du sentir. Prenons deux exemples : 
[25] Entoure les mots qui te semblent correspondre à ce que tu ressens. Complète les (sic) avec tes propres mots : Joie Colère Douceur Tristesse Chagrin Rage Peur Laideur Ennui Amour Beauté Lâcher-prise

[26] Voler est un des plus vieux rêves de l'humanité. Voler, c'est s'évader, s'échapper du quotidien, libérer son corps. Toi aussi en jouant avec ta famille ou tes copains, tu connais cet état de lâcher-prise, par exemple, quand tu fais de la balançoire, du trampoline, quand, plus jeune, tes parents te faisaient sauter dans leurs bras ou quand tu rêves. Te rappelles-tu la sensation éprouvée à ce momentlà ? Peux-tu la décrire?

Comme dans le livret précédent, les commentaires font appel aux réactions émotives de l'enfant, à son expérience [26], mais ici, il s'agit d'aider l'enfant à préciser ce qu'il perçoit et de le mettre en mots. D'après S. Chaumier (2010), cette approche de l'art par les sens est en voie de généralisation dans la médiation de l'art contemporain. On citera une dernière description-commentaire (plutôt longue), et les quatre activités consécutives (une sorte de "jeu du téléphone arabe ») afin de mieux en résumer la démarche :

[27] Dans la Salle 3, les installations vidéo Alamo et Color (« couleur» en anglais) ont été filmées au Texas (États-Unis). Le Texas est un État pluri-culturel constitué par différentes communautés (amérindiens, anglo-saxons, hispaniques, afroaméricains) au fil de l'Histoire. Il a appartenu au Mexique, puis est devenu indépendant et a finalement été rattaché aux États-Unis. Le cinéma a contribué à associer le Texas à l'image du cow-boy.

$\rightarrow$ Dans Alamo, les personnes qui relatent l'histoire de Fort Alamo nous proposent la version de chaque communauté. La transmission de l'histoire par une seule communauté l'ayant vécu est insuffisante pour comprendre tous les enjeux.

$\rightarrow$ Dans Alamo, Sylvie Blocher montre que les personnes ont souvent plusieurs versions d'un même événement historique. Tu vas expérimenter la transmission d'une histoire et constater que ta mémoire et celle de ton entourage peut aussi modifier le sens de l'histoire originale.

Demande à une médiatrice de te raconter l'histoire d'Alamo.

De mémoire, tu vas communiquer cette histoire à une personne qui t'accompagne. Ellemême peut la transmettre à d'autres, si vous êtes plusieurs. La dernière personne ayant entendu l'histoire doit la restituer à une médiatrice.

Tu peux écrire la dernière version. Que remarques-tu?

Le commentaire s'apparente à une tâche, telle qu'on la trouve dans la pédagogie actionnelle ( « tu vas expérimenter »). Rappelons que celle-ci considère l'enfant comme un acteur social, et qu'on ne communique pas seulement pour parler avec l'autre, mais aussi pour agir sur l'autre. Aussi, les activités développent les compétences langagières (oral/écrit), les compétences communicatives (« demander à une médiatrice/raconter l'histoire ») mais c'est bien l'action finale («Que remarques-tu ?») à laquelle aboutit l'activité qui va permettre à l'enfant de comprendre les aléas de «la transmission de l'Histoire » et donc de donner un sens à l'œuvre.

\section{L'ouverture interculturelle}

Conformément au propos de l'exposition, l'accent est mis sur l'appréhension de la diversité du monde et des langues ${ }^{23}$. La dimension interculturelle est mise en avant dès la couverture du cahier, à travers la métaphore du voyage ${ }^{24}$ qui est aussi une découverte de la pluralité des cultures et des langages : 
[28] L'exposition va te faire voyager aux quatre coins du monde [...]. Tu vas découvrir des histoires du monde. Tu vas entendre différentes langues, voir s'exprimer différents langages, ceux du corps, de la voix, des mots. complexité de la tâche se devine dans certaines activités à la fois cognitives, perceptives et langagières. En dépit d'une syntaxe relativement simple, certains mots peu courants apparaissent çà et là («au fil de ", « sévit » lus plus haut) qui pourraient exiger une explication de l'adulte; quelques activités sont peu intuitives: ainsi, distinguer la "tristesse» du «chagrin» n'est pas chose facile ([25]), et l'idée de «lâcher-prise » parait bien compliquée pour un enfant ([26]) : que peut signifier, pour lui, se libérer des aliénations, du travail, du train-train quotidien? Toujours dans les exemples [25] et [26] : un enfant a-t-il les moyens de mettre en mots spontanément ses émotions ? La réussite de ces activités, nous semble-t-il, est tributaire de l'aide de l'adulte accompagnateur, qui pourra toutefois trouver les réponses justes puisque le texte n'est pas basé sur les connaissances artistiques ou savantes. Il pourra, de ce fait, aisément élargir le propos, impliquer l'enfant et l'amener à un jugement critique.

Pour toutes ces raisons, le livret est davantage adapté à la famille qu'à l'enfant seul, quand bien même l'adulte participerait plus à un contexte "périlectoral» que «lectoral» (Prince, 2009, p. 11) comme dans certains livres de jeunesse. Pour conclure, ce livret donne accès à une vision élargie du monde et, quoique difficile par endroits, il valorise l'enfant-visiteur en lui permettant d'affiner sa perception des choses et donc d'acquérir un autre type de savoir. 


\section{L'expérience à travers le jeu : inventer d'autres liens avec et dans le musée}

\section{Du musée comme espace de jeu...}

\section{la typographie grasse et quelque peu froide, la sobriété du noir et blanc qu'égayent de} rares reproductions colorées lui confèrent à la fois lisibilité et caractère, mais renforcent sa connotation élitiste. Bien que le format en rende le feuilletage aisé, les pages ne contiennent pas de titre et la numérotation («15|V102|») est mystérieuse. On se demande d'abord s'il vise un public enfant. En consultant le site web du musée, on apprend que ce « carnet de jeu » a été conçu par deux artistes. Et c'est effectivement de jeu qu'il s'agit. Voyez plutôt :

[30] Fais la visite d'une des salles du musée à reculons.

[31] Repère les caméras de surveillance. Réalise une performance de 30 secondes maximum en espérant que quelqu'un te regarde.

[32] Choisis une seule œuvre. Organise le concours de celui qui la regarde le plus longtemps.

[33] 1. Regarde une œuvre. 2. Frotte-toi les yeux pendant 5 minutes. 3. Regarde à nouveau l'œuvre.

[34] Si ton hygiène corporelle te le permet, retire tes chaussures pour regarder les œuvres.

[35] Choisis une œuvre puis décide de la bande son qui va avec.

[36] Va dans le jardin et crie le nom de ton artiste préféré.

[37] Trouve un moyen de toucher une œuvre sans que ton corps ne rentre en contact avec.

[38] Assieds-toi là où tu te trouves actuellement et prends une pause bien méritée. 
dans son acception commune) qui puisse indiquer un savoir sur la collection. Du coup, les activités pourraient se faire dans n'importe quel musée d'art contemporain. Ce n'est pas seulement le livret qui est un support de jeux, mais tout le musée. Une question surgit : un tel livret ne ramène-t-il pas, peu ou prou, le musée à un espace de loisir ?

\section{... au jeu de l'art contemporain et ses codes}

La volonté de faire vivre à l'enfant une expérience dans une atmosphère réjouissante peut cependant faire œuvre de culture. En effet, une lecture plus attentive montre que les jeux le poussent à acquérir une familiarité avec les règles de l'art contemporain, à lui transmettre les bons réflexes, bref, à favoriser une "littératie muséale " (Poli \& Triquet, 2003) :

[39] Regarde attentivement les œuvres pour voir comment elles sont accrochées.

[40] Trouve une bonne position pour regarder un tableau.

Les concepteurs tiennent avant tout à faire apprendre des habiletés, des modalités de réactions face à l'art qui vont de l'humour [35] au sérieux, comme dans les exemples sus-cités ou dans le jeu suivant qui permet à l'enfant de prendre conscience de son état de regardeur, comme le veut la création contemporaine :

[41] Regarde une œuvre de la collection et demande-toi si ton voisin voit la même chose que toi.

Les jeux invitent l'enfant à entrer dans le monde de l'art contemporain, implicitement ; on sait en effet les difficultés à penser la question du sens de certaines œuvres, de «ce que l'artiste a voulu dire » et de le transmettre en discours. On citera deux derniers jeux, qui ont en commun d'élargir au-delà du beau le panel des effets de l'expérience esthétique :

[42] Cherche l'œuvre qui pour toi est la plus laide du musée et reproduis-la le plus fidèlement possible ci-dessous.

[43] Sens une œuvre.

Le premier le fait sur un mode ludique et détourné, puisqu'en principe, on recopie l'œuvre la plus belle, le second ([43]) plus sérieusement. Prenons par exemple le tableau d'un bouquet de fleurs intitulé «L'odeur est une forme qui ne se voit pas " (Présence Panchounette), au-dessus duquel est posé un diffuseur de parfum «moyenne gamme » ${ }^{26}$. L'enfant qui fera jouer ses sens, dans le cas présent l'odorat, n'appréciera probablement guère... Ce faisant, il pourra déchiffrer l'un des principes de fonctionnement de l'art contemporain, à savoir que celui-ci n'est pas seulement lié au plaisir, mais qu'il est ouvert à toutes les interprétations possibles, à toutes les réactions, y compris le dégout! Ce qui importe, c'est bien que le jeu suscite une réception active, une réaction verbale ou non-verbale de l'enfant. La question est alors la suivante: pourra-t-il passer outre cette première perception innocente? Question connexe: qu'advient-il en cas de réaction "négative " («ce n'est pas de l'art») ? C'est là que réside le point critique : entre le moment où l'enfant dit «Beurk !» et le moment où il reprend son chemin, le projet d'acculturation peut rester lettre morte sans l'encadrement bienveillant de l'adulte, sans un encouragement à exprimer et à partager son appréciation. 


\section{Le livret pour famille : un enrichissement interpersonnel ?}

69 À ce point de notre analyse, il nous a paru utile de mettre en perspective le livret qui a précédé et le livret pour famille publié par le Mac/Val : qu'apporte-t-il de plus?

70 À première vue, ce dernier s'adresse à la famille : la mention «Familles Enfants » en couverture, le «mode d'emploi » sur lequel s'ouvre le document, dont les règles sont valables "pour tous", ainsi que la marque énonciative "vous » engagent une lecture partagée de l'enfant et de l'adulte :

[44] Mode d'emploi

Les étapes de ce jeu peuvent se faire dans l'ordre que vous voulez. À vous de jouer !

Pour tous: n'oubliez pas qu'en toutes circonstances, la sécurité des œuvres ainsi que

la tranquillité des autres visiteurs doivent être respectées. Comme dans tout musée,

il est interdit de toucher les œuvres! Mais à part cela, n'hésitez surtout pas à expliquer que dans le musée chacun a le droit à la curiosité, à l'imagination, à l'expression et aux émotions!

71 En réalité, il s'adresse aux adultes, comme l'indiquent deux indices. Le premier est constitué par le verbe "expliquer» qui implicitement définit une médiation « verticale » puisque le livret attribue à l'adulte un rôle d'enseignant, plutôt que celui de compagnon de visite, et place l'enfant comme inférieur dans l'ordre du savoir. Second indice : la description succincte (le nom propre, le geste créatif) qui manifeste le retour à une approche frontale de l'œuvre. On en donnera pour preuves deux exemples :

[45] Voyage - voyage

Kimsooja est une artiste nomade. Elle promène dans le monde sa silhouette habillée de noir. Voyageuse immobile et silencieuse, elle parcourt villes et campagnes d'Asie, d'Amérique ou d'Europe, comme dans un rêve pourtant bien réel. Sa figure évoque l'exilé, l'étranger ou l'ambassadeur d'une contrée lointaine. L'artiste passe par Paris mais son voyage continue. Quelle sera sa prochaine destination ? À vous de dessiner le prochain paysage qu'elle va traverser.

[46] Signez ici

Le duo d'artistes Nøne Futbol Club s'est approprié ces deux estampes de Pablo Picasso par un geste discret et pourtant brutal : ils les ont taguées et re-signées ! À vous de rayer le nom de Picasso et d'inventer votre signature d'artiste à la place.

Bien que le livret ne nécessite, là aussi, aucun prérequis (courants artistiques, noms propres, normes, citations, etc.), il suppose tout de même une certaine familiarité avec le monde de l'art contemporain et ses discours comme en témoigne l'exemple [46], où affleurent des manières de dire typiques de la critique d'art qui rendent le commentaire hors d'atteinte pour un enfant.

Quant aux activités (essentiellement du dessin), elles sont toutes destinées à l'enfant. Bien qu'elles mettent en avant l'imagination de l'enfant, on note toutefois qu'elles sont placées sous le regard parental, dans la mesure où il revient à l'adulte de faire le lien entre l'œuvre in situ et l'activité ludique proposée. Seule l'activité portant sur le paysage de bord de mer photographiée par A. Varda peut éventuellement être partagée (" seul ou à plusieurs ») :

[47] seul ou à plusieurs, créez l'image de fin du film en prenant la pose devant l'œuvre. Ex: un baiser d'adieu, des retrouvailles inespérées... Si vous pouvez, prenez-la en photo et envoyez-la à l'adresse publicsmusee@macavl.fr. Nous publierons les plus émouvantes sur le site. 
74 C'est pourquoi il parait peu probable que le livret préfigure un espace de lecture et d'échange au sein de la famille. Sans doute les livrets analysés dans nos troisième et quatrième parties y étaient plus propices. Du coup, on se demande si le jeu représente un outil de partage, où s'il permet tout simplement d'occuper l'enfant pendant que les grands « regardent »... Le livret résout la bivalence des destinataires en alternant les passages adressés à l'adulte et les moments consacrés aux enfants en deux espaces distincts. Au bout du compte, il montre que c'est toujours l'enfant qui accompagne l'adulte, alors que l'on pouvait légitimement supposer que, grâce au livret, il aurait pu enfin ! jouer le rôle d'accompagnateur.

\section{Conclusion : vers un accès à l'art pour « un public enfant le plus large »?}

Ces livrets ont tous en commun, à des degrés divers, de "faire apprendre en s'amusant», de favoriser la compréhension, l'expression, l'épanouissement, l'autonomie de l'enfant, que ce soit par le regard, le ludique, les sens ou l'expérience... Certes, il n'y a guère de similitudes entre ces approches de la médiation et pourtant, une évolution générale se profile, peut-être, dans la mesure où la plupart des livrets tendent à valoriser le plaisir de visiter, la relation avec l'accompagnateur ou le musée, le ludique comme autant de modalités pour une meilleure appropriation de l'exposition. C'est sur ce point que l'on terminera, par deux remarques portant sur les formes de médiation et sur l'accès à l'art qu'elles présupposent.

Premièrement, on pourrait penser que ces approches, telles que présentées ici, soient exclusives les unes des autres. Or, il nous semble que les différences entre les diverses formes de médiation ne sont pas irréductibles, et qu'elles pourraient parfaitement se compléter, y compris dans un même livret. Pour s'en tenir au ludique, on l'a vu, celui-ci reste au service d'une visée éducative et du contenu, non l'inverse. C'est la raison pour laquelle on ne saurait partager le pessimisme de certains chercheurs ou médiateurs qui ne voient dans le ludique qu'une soumission au paradigme du divertissement ${ }^{27}$. On souscrit donc pleinement à la position de S. Chaumier (2010) lorsqu'il défend des " voix mixtes ", en plaidant pour une approche qui mêle le didactique, l'expérientiel, les cultures et le ludique.

Deuxièmement, il faut dire que si la plupart des livrets se proposent de donner les clés pour initier à l'art, beaucoup offrent en réalité des clés pour entrer dans un monde d'initiés ${ }^{28}$. Dès lors, comment se donner véritablement les moyens de démocratiser l'art, pour que le leitmotiv "diffuser l'art en direction d'un public enfant» qu'entonnent les institutions muséales (y compris les musées labellisés «Musées de France ») ne reste pas un vœu pieux?

Un élément de réflexion, sinon de réponse, se trouve selon nous dans la question de la langue. Ce que mettent finalement en avant ces livrets, à de rares exceptions près, c'est cet attachement passionnel et tout francophone à la langue soutenue, mais qui peut présenter un facteur inhibiteur - ce que les musées semblent faire mine d'ignorer. Les études à visée évaluative l'ont pourtant montré il y a déjà quelques années : les enfants sont tout aussi exigeants envers le contenu que les adultes (Duclos, Philippeaux \& Poli, 2010), mais sans doute peut-on leur fournir les informations dans une parole plus simple, davantage dépouillée de littérarité et de technicité, plus propice à leur gout, à 
leur âge (Rigat, 2012). Rappelons avec M. Bakhtine (1977, p. 124) que «le mot est le territoire commun du locuteur et de l'interlocuteur ». La langue est un levier de démocratisation, et une langue plus simple ne saurait signifier une mise en veille des exigences éducatives et culturelles. Plutôt, il reste à concilier des limites dues à l'image que se font les institutions du visiteur modèle (Davallon, 2011), aux habitudes institutionnelles, à la prégnance des représentations. Cela nous semble important, si l'on ne veut pas que le public familial demeure une simple cible marketing, comme il apparait pour l'instant dans les travaux récents (Merleau-Ponty, Davallon \& Caillet, 2016, p. 24).

\section{BIBLIOGRAPHIE}

BAKHTINE, M. (1977) [1929]. Le marxisme et la philosophie du langage. Essai d'application de la méthode sociologique en linguistique. Trad. du russe par M. Yaguello. Paris : Éd. de Minuit.

Barbieux, C. (2011). « Évaluer les effets des dispositifs ludiques destinés aux enfants en visite familiale ». La Lettre de l'OCIM 135, p. 19-26. En ligne : http://journals.openedition.org/ocim/870. BROUGÈRE, G. (2012). « La culture matérielle enfantine entre le cute et le cool ». Strence 4. En ligne : http://journals.openedition.org/strenae/776.

CAILLET, É. (1994). «L'ambiguïté de la médiation culturelle : entre savoir et présence ». Culture et Musées 6, p. 53-73. En ligne : http://www.persee.fr/doc/pumus_1164-5385_1994_num_6_1_1046. CHAUMIER, S. (2010). «La muséographie de l'art, ou la dialectique de l'œuvre et de sa réception ». Culture et Musées 16, p. 21-43. En ligne : http://www.persee.fr/doc/ pumus_1766-2923_2010_num_16_1_1558.

CHAUMIER, S. (2011). « La nouvelle muséologie mène-t-elle au parc? ». In : Chaumier, S. (dir.), Expoland. Ce que le parc fait au musée : ambivalence des formes de l'exposition. Paris : Éd. Complicités, p. 65-88.

DAVAllon, J. (2011). « Le pouvoir sémiotique de l'espace. Vers une nouvelle conception de l'exposition? » Hermès 3, 61, p. 38-44. En ligne : https://www.cairn.info/revue-hermes-larevue-2011-3-page-38.htm.

DAVAllon, J. (2000). L'Exposition à l'œuvre. Stratégies de communication et médiation symbolique. Paris : L'Harmattan.

DESVAllÉEs, A. \& MAIRESSE, F. (2011). Dictionnaire encyclopédique de muséologie. Paris : A. Colin. Duclos, A., PHILIPPEAUX, F. \& POLI, M.-S. (2010). « De l'utilité du "texte enfant" au musée ». La Lettre de l'OCIM 132, p. 28-33. En ligne : http://journals.openedition.org/ocim/386.

DUFIET, J.-P. (2013). «L'art écrit dans le Guide de Musée : l'exemple du guide du Louvre ». In : Barkat-Defradas, M. \& Benoist, S. (dirs), Comment parler de l'art? Approches discursives et sémiotiques. Paris : CNRS Éd., p. 13-40.

EIDELMAN, J. \& JONCHERY, A. (2011). « Sociologie de la démocratisation des musées ». Hermès 3, 61. p. 52-60. En ligne : https://www.cairn.info/revue-hermes-la-revue-2011-3-page-52.htm. 
GARDES TAMINE, J. (2004). Pour une grammaire de l'écrit. Paris : Belin.

GLICENSTEIN, J. (2012). « Éditorial ». Marges 15, p. 5-7.

GLICENSTEIN, J. (2008). « L'art contemporain peut-il être populaire ? Remarques à propos de Nuit blanche ». Nouvelle revue d'esthétique 1, p. 21-28.

GOB, A. \& MONTPETIT, R. (2010). «Introduction ». Culture et Musées 16, p. 13-19.

GROS, F. (2009). Marcher, une philosophie. Paris : Carnets Nord.

JACOBI, D., MEUNIER, A., ROMANO, S. (2000). « La médiation culturelle dans les musées : une forme de régulation sociale ». Recherches en communication 13, p. 37-60. En ligne : http://sites.uclouvain.be/ rec/index.php/rec/article/viewFile/2701/2501.

JACOBI, D. \& COPPEY, O. (1995). «Introduction - Musée et éducation : au-delà du consensus, la recherche du partenariat ». Publics et Musées 7, p. 10-22. En ligne : http://www.persee.fr/doc/ pumus_1164-5385_1995_num_7_1_1053.

JONCHERY, A., BIRAUD, S. (2014). « Musées en famille, familles au musée. De l'expérience de visite des familles à des politiques muséales spécifiques ». Informations sociales 181, p. 86-95. En ligne : https://www.cairn.info/revue-informations-sociales-2014-1-page-86.htm.

LE MAREC, J. (2011). « L'environnement et la participation au musée : différentes expressions culturelles des sciences ». Hermès 3, 61, p. 167-174. En ligne : https://www.cairn.info/revuehermes-la-revue-2011-3-page-167.htm.

LE MAREC, J. (2006). «Les musées et bibliothèques comme espaces culturels de formation ». Savoirs 2, 11, p. 9-38. En ligne : https://www.cairn.info/revue-savoirs-2006-2-page-9.htm.

LESAFFRE, G., WATREMEZ, A. \& FLON, É. (2014). « Les applications mobiles de musées et de sites patrimoniaux en France : quelles propositions de médiation? » La Lettre de l'OCIM 154, p. 5-13. En ligne : http://journals.openedition.org/ocim/1423.

MICHAUX, Y. (1989). « Voir et ne pas savoir ». Les cahiers du Musée national d'art moderne 17-33, p. 25. остовRE, S. \& SIROTA, R. (2013). «Introduction. L'enfance au prisme de la culture : approches internationales ». In : Enfance et Cultures sous le regard des sciences sociales. Paris : Ministère de la Culture et de la Communication, Paris Descartes, $9^{\mathrm{e}}$ journée de sociologie de l'enfance, p. 17-31.

MERLEAU-PONTY, C., DAVAllon, J., CAILlET, E. (2016). « Il était une fois... ». In : Serain, F. et al. (dirs), La médiation culturelle. Cinquième roue du carrosse ? Paris : L'Harmattan, p. 13-28.

POLI, M.-S. (2013). «Éducation et musée ». Culture et Musées, Hors-série La Muséologie : 20 ans de recherches, p. 165-187.

POLI, M-S. \& TRIQUET, É. (2003). «Écrire au muséum : pour une approche résolument transdisciplinaire ». LIDILEM 23, p. 45-68.

PRINCE, N. (dir.) (2009). La littérature de jeunesse en question(s). Rennes : Presses universitaires de Rennes.

RIGAT, F. (2012). Écrits pour voir. Aspects linguistiques du texte expographique. Turin : Trauben.

TURIN, J. (2003). « La littérature de jeunesse et les adolescents. Évolution et tendances ». BBF 3 , p. 43-50. En ligne : http://www.enssib.fr/bibliotheque-numerique/documents/35714-lalitterature-de-jeunesse-et-les-adolescents.pdf. 
WILFRIED, L. (2007). « L'autonomie enfantine à l'épreuve des “surdoués”. Contribution ethnographique à une approche sociale de l'enfance ». L'Homme et la société 3, 165-166, p. 205-221. En ligne : https://www.cairn.info/revue-l-homme-et-la-societe-2007-3-page-205.htm.

\section{Corpus d'étude}

Parcours enfant, Trop grand! (2008). Paris : Le Louvre.

Livret Jeune public, Les Papesses. Avignon : Fondation Lambert, du 90/06/2013 au 11/11/2013.

Cahier du musée. (2015). Musée d'art contemporain du Val-de-Marne.

Enfants Familles, Bon plan, L'effet vertigo, Collection du musée, Musée d'art contemporain du Valde-Marne, à partir du 24/10/2015.

Cahier découverte enfant, S'inventer autrement. Sylvie Blocher. Sète : Centre régional d'art contemporain, du 23/10 /2015 au 31/01/2016.

\section{NOTES}

1. C'est ce qu'ont affirmé les médiateurs lors des formations organisées par l'office de coopération et d'information muséales (Ocim) autour de la médiation en direction des enfants. Nous tenons à remercier les organisatrices des journées auxquelles nous avons participé durant les années 2011-2013, S. Le Berre, A. Fourrès, E. Maczek, ainsi que les nombreux participants pour les échanges fructueux et auxquels ces pages doivent beaucoup.

2. « Mythes fondateurs. D’Hercule à Dark Vador », du 17 octobre 2015 au 4 juillet 2016.

3. On fait ici allusion ici à la visite comme outil de culture telle que théorisée par J. Davallon (2000).

4. Notre objet n'étant pas de nous livrer à une réflexion sur l'éducation au musée, on renverra sur cette question à D. Jacobi, O. Coppey (1995), D. Jacobi, A. Meunier, S. Romano (2000) et, pour une mise au point exhaustive, à M.-S. Poli (2013).

5. Selon la Convention des droits de l'enfant de 1989, est considéré comme tel une personne âgée de moins de 18 ans.

6. Cette tranche d'âge varie en réalité selon les institutions; dans tous les cas, il s'agit d'enfants déjà lecteurs.

7. Selon l'expression de J. Turin (2003).

8. Comme le souligne B. Meyerson (2006), «It is important to remember that museum visiting families often contain multiple children that can span a variety of ages and abilities ». In : Quelles expositions d'art pour les enfants? Colloque Partages, Les Journées professionnelles du Louvre, 28 avril 2006.

9. Notre approche discursive ne nous permet pas d'évaluer l'usage effectif du document: le travail d'A. Duclos, F. Philippeaux et M.-S. Poli (2010) en a cependant soulevé la diversité et la multiplicité.

10. L'expression est empruntée à J. Galard (in Chaumier 2010, p. 28).

11. Dans sa première acception, s.v. Promenade «Action de se promener, d'aller à l'extérieur pour se divertir» (Trésor de la langue française informatisé). L'italique est nôtre dans tous les exemples et citations.

12. La " promenade » est un cliché de la littérature de visite au Louvre, et renvoie à un " artifice mondain », pour le dire comme F. Gros (2009), bien parisien.

13. s.v. Promenade : «Au fig. Cheminement mental, intellectuel, spirituel.» ; s.v. Parcours : « $A u$ fig., PÉDAG., RELIG. CATH. Parcours (de catéchèse, catéchétique). Programme pédagogique annuel de 
cheminement de la foi à parcourir par les enfants d'âge scolaire avec leurs parents, leurs catéchistes ; p.méton., recueil (de fiches), manuel exposant ce programme.» (Trésor de la langue française informatisé).

14. Pour Y. Michaux (1989, p. 25), « la vision a longtemps été et reste le paradigme de la clarté du savoir ».

15. J.-P. Dufiet (2013) en arrive aux mêmes conclusions dans son analyse du guide du musée.

16. Précisons toutefois que cela est plus vrai encore dans d'autres documents publiés par le Louvre et par d'autres musées nationaux.

17. Notons que les images reproduisent aussi des œuvres de la culture cultivée: H. Matisse, Ingres, L. de Vinci, etc. Toutes les références sont disponibles en fin de livret, dans la table des illustrations.

18. Les récits sont en outre soigneusement choisis par rapport au texte adulte: il n'y a pas de mention de la tentative de suicide de L. Bourgeois par exemple, ou encore d'allusion au mariage pour tous...

19. C'est ce qui explique les réactions tièdes, sinon hostiles observées chez de nombreux médiateurs lors des séminaires que nous avons tenus (cf. note 1).

20. Et forger, en même temps, l'ethos d'un musée jeune, vivant, dynamique.

21. Mais on soupçonnait déjà une voix féminine, vues les marques énonciatives (le nous dans l'exemple [17]) qui unissent l'énonciateur et le lecteur par le sexe, ce qu'a confirmé la liste des conceptrices en dernière page du livret.

22. Malgré un adjectif au masculin que l'on ne s'explique pas : «Tu verras peut-être quand tu seras plus grand le célèbre film qui raconte la vie de Camille Claudel ».

23. La diversité des corps (l'installation « Change the scenario » mettant en scène un albinos) n'a pas été retenue dans le livret pour enfants.

24. Le voyage dans le temps et l'espace est un topos dans les livrets pour enfants. Voir F. Rigat (2012).

25. Au passage, on note que la glose explicative est bien moins politiquement correcte sur le plan connotatif que la définition dictionnairique du Petit Robert, s.v. Favela : «Au Brésil, Ensemble d'habitations populaires de construction sommaire et dépourvues de confort. »

26. Voir sur le site du musée: http://www.macval.fr/IMG/pdf/Presence_Panchounette__Laetitia_Chauvin_BD.pdf.

27. Cette position correspond assez bien à ce qu'on trouve chez F. Brugères : "Face à l'injonction démocratique du "divertissez-vous !", le musée ne peut opposer qu'un "ennuyez-vous au musée" ou un "divertissez-vous au musée". » (in Chaumier, 2011, p. 85).

28. Voir à ce propos J. Glicenstein (2008).

\section{RÉSUMÉS}

Notre article aborde le rôle et la conception des livrets publiés par les musées d'art et destinés à toute la famille ou aux enfants hors temps scolaire, dans le cadre de loisirs donc. Notre objectif est d'explorer comment ces outils d'aide à la visite s'inscrivent dans une conception plus culturelle de l'éducation au et par le musée et comment ils prennent en compte l'enfant dans sa dimension socioaffective. Notre point de vue, discursif et énonciatif, nous permettra d'observer ce qui se construit entre les œuvres, l'enfant et l'adulte et, plus largement, entre l'enfant et le 
musée. Ces questions, illustrées par l'analyse de cinq livrets publiés récemment, seront mises en relation avec un questionnement bien ancien, mais loin d'être résolu, sur la démocratisation de l'art que ces documents suggèrent. À travers la diversité des partis pris, on repère néanmoins une évolution générale, dans la mesure où la plupart des livrets tendent à valoriser le plaisir de la visite, la relation avec l'accompagnateur ou le musée, le ludique comme autant de modalités pour mieux s'approprier l'exposition, pour faire de la visite un «loisir éducatif» (l'edutainment d'outre-Manche), un plaisir par conséquent lié à la culture, à l'expérience, à la sociabilité - pas seulement à la contemplation.

This article analyzes the Art Museum Guide for Children with Parents or Caregivers, a publication addressed to families or to children engaged in post-school activities. Our aim is to understand how these types of handbooks can help visitors to relate to culture in a wide perspective - how they develop an awareness of what the museum is and what it is for - and how these manage to take the child's socioaffective needs into full account. By means of a discursive and "enunciative" approach, we investigate the dynamics of the relationship between children and paintings, children and adults, as well as of that between the child and the museum. Such topics, as illustrated through five recently published books, will be linked to the time-old (yet never fully resolved) question of the democratization of art. Our analysis will evidence a tendency towards an increase in the pleasant aspect of the visit, which is resembling more and more an edutainment, leaving aside the mere aspect of contemplation of beauty.

\section{INDEX}

Mots-clés : exposition, livret pour enfant, médiation, culture, loisir

Keywords : exhibition, children's booklet, mediation, culture, leisure

\section{AUTEUR}

\section{FRANÇOISE RIGAT}

Université de la Vallée d'Aoste, IT-11100, Italie 